\title{
Multiscale Computational Design of Core/Shell Nanoparticles for Oxygen Reduction Reaction
}

\author{
Zhengzheng Chen, ${ }^{\circ} \mathrm{Xu}$ Zhang, and Gang Lu* \\ Department of Physics and Astronomy, California State University, Northridge, California 91330, United States \\ Supporting Information
}

ABSTRACT: We propose a multiscale computational framework to design core/shell nanoparticles (NPs) for oxygen reduction reaction (ORR). Essential to the framework are linear scaling relations between oxygen adsorption energy and surface strain, which can be determined for NP facets and edges from first-principles and multiscale QM/MM calculations, respectively. Based on the linear scaling relations and a microkinetic model, we can estimate ORR rates as a function of surface strain on core/shell NPs. Employing the multiscale framework, we have systematically examined the ORR activity on Pd-based core/shell NPs as a function of their shape, size, shell thickness, and alloy composition of the core. Three NP shapes-icosahedron, octahedron, and truncated octahedron-are explored, and the truncated octahedron is found to be the most active and the icosahedron is the least active. $\mathrm{Ni}_{x} \mathrm{Pd}_{1-x} @ \mathrm{Pd} \mathrm{NPs}$

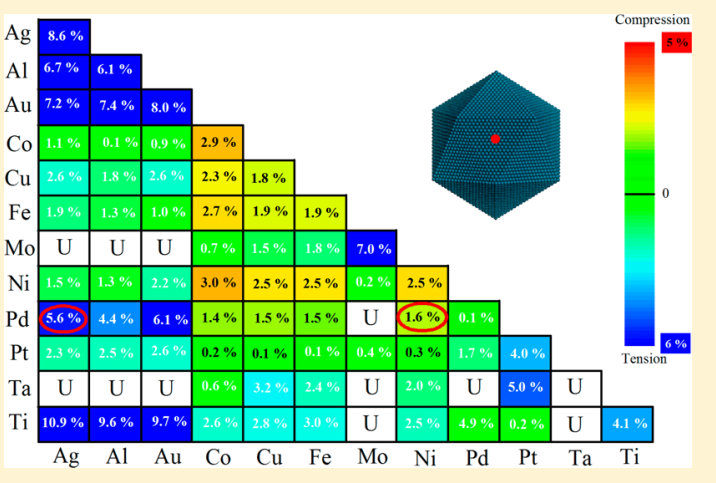
with high $\mathrm{Ni}$ concentrations and thin shells could exhibit higher ORR rates than the pure Pt(111) surface and/or Pt NPs. $\mathrm{Ag}_{x} \mathrm{Pd}_{1-x} @ \mathrm{Pd}$ in the truncated octahedron shape and high $\mathrm{Ag}$ concentrations are predicted to be even more active than $\mathrm{Ni}_{x} \mathrm{Pd}_{1-x} @ \mathrm{Pd} \mathrm{NPs}$ under the same conditions. The highly active $\mathrm{Ag}_{x} \mathrm{Pd}_{1-x} @ \mathrm{Pd}$ and $\mathrm{Ni}_{x} \mathrm{Pd}_{1-x} @ \mathrm{Pd} \mathrm{NPs}$ are thermodynamically stable.

\section{INTRODUCTION}

Past decades have witnessed tremendous progress in the development of novel nanostructured catalysts for energy conversion and storage. In particular, multimetallic core/shell nanoparticles (NPs) have emerged as one of the most attractive candidates for highly efficient, stable, and scalable catalysts owing to their unique morphological, electronic, and chemical properties as compared to their bulk counterparts. ${ }^{1-13}$ These properties depend on the size, shape, shell thickness, and chemical composition of the core/shell NPs and can be tuned to provide optimal combinations of activity, selectivity, and stability for particular catalytic reactions. ${ }^{14-18}$ For example, the size of NPs determines the number of active surface sites, while the shape of NPs dictates which facets are exposed or available for catalytic reactions. In multimetallic core/shell NPs, more complex mechanisms may come into play. For instance, surface strain due to the lattice mismatch between the core/shell materials could significantly alter the surface properties. ${ }^{19-21}$ The d-band center of the metallic shell may be shifted in the presence of the metallic core, thanks to the combination of ligand and strain effects, ${ }^{22-25}$ which can be controlled by the chemical composition and the shell thickness of the NPs. ${ }^{26-28}$ Obviously, all these factors-size, shape, shell thickness, and chemical composition-are intertwined, and are collectively responsible for the catalytic performance of the core/shell NPs. However, the interplay between these factors is highly complex and needs to be unraveled. In particular, how these factors conspire to influence catalytic activities in electrochemical reactions has yet to be established. As a result, we face significant hurdles in experimental optimizations of core/shell catalysts because there are too many materials parameters to be explored by trial and error. Therefore, there is a critical need in theoretical understanding and computational design that would accelerate the discovery and development of superior core/shell nanocatalysts.

In this work, we take the challenge by proposing a multiscale computational framework that integrates these factors into the design of core/shell NPs for optimal catalytic activities. Although the computational framework is general, we focus on oxygen reduction reaction (ORR) as an example to demonstrate its application to electrocatalysis. ORR is a key chemical reaction in proton exchange membrane fuel cells and metal-air batteries. ${ }^{29-32}$ At present, the best ORR catalysts consist exclusively of $\mathrm{Pt}$ and suffer from deficiencies such as sluggish ORR kinetics and high material costs, preventing them from being widely deployed in large-scale applications. Multimetallic core/shell NPs are among the most promising alternatives to Pt-based catalysts. Here, we employ the multiscale computational strategy to design Pd-based core/ shell NPs that are potentially less expensive and more active than $\mathrm{Pt}^{33,34}$ To this end, we have systematically examined the ORR activity on Pd core/shell NPs as a function of their shape,

Received: November 10, 2016

Revised: December 28, 2016

Published: January 6, 2017 
size, shell thickness, and alloy composition of the core. Detailed analysis is performed to unravel the interplay between these factors. The thermodynamic stability of the core/shell NPs is also examined for the highly active catalysts. Previous work has revealed a linear scaling relation between surface strain and oxygen adsorption energy-a descriptor for ORR activity. ${ }^{35}$ Thus, the surface strain on the NPs is the focus of this study which sets our work apart from similar studies. By combining a microkinetic model with multiscale quantum mechanics/ molecular mechanics (QM/MM) and first-principles calculations, we can assess ORR activity on the core/shell NPs. Based on the multiscale framework, we can computationally design Pd-based core/shell NPs with superior ORR activity to Pt.

\section{MULTISCALE COMPUTATIONAL STRATEGY}

In a number of influential papers, Nørskov and collaborators have shown that there are universal linear relations between activation energies and adsorption energies of reaction intermediates for a class of catalytic reactions, ${ }^{36}$ including ORR, which stem from the similarity among the transition state structures and the existence of Brønsted-Evans-Polanyi-type scaling relations. In particular, the oxygen adsorption energy $E_{\mathrm{O}}$ was identified as a descriptor for overall ORR activity on both pure metal and alloy surfaces. ${ }^{37,38}$ If the binding between oxygen and the metal surface is too strong, the bottleneck of ORR is the removal of adsorbed $\mathrm{O}$ and $\mathrm{OH}$ species. Conversely, the bottleneck is the dissociation of $\mathrm{O}_{2}$ on the metal surface; both rate-limiting processes can be characterized by $E_{\mathrm{O}}$. The overpotential of ORR is defined as the minimal electrode potential at which the overall cathode reaction, i.e., the coupled proton and electron transfer to the adsorbed oxygen or hydroxide, is at equilibrium, thus directly connected to $E_{\mathrm{O}}$. Moreover, if the ORR activity is plotted as a function of $E_{\mathrm{O}}$, one obtains a volcano plot that agrees very well with the experimental measurements. ${ }^{38,39}$

More specifically, the linear relationships between the activation energy, $E_{a}$ of the ORR rate-limiting reactions and $E_{\mathrm{O}}$ can be written as

$$
E_{\mathrm{a}}= \begin{cases}-0.616 E_{\mathrm{O}}(\varepsilon)-0.67 & E_{\mathrm{O}}(\varepsilon)<-1.09 \mathrm{eV} \\ 1.8 E_{\mathrm{O}}(\varepsilon)+1.96 & E_{\mathrm{O}}(\varepsilon) \geq-1.09 \mathrm{eV}\end{cases}
$$

The coefficients in eq 1 are taken from Nørskov et al. ${ }^{39}$ When $E_{\mathrm{O}}(\varepsilon)<-1.09 \mathrm{eV}$, the rate-limiting process is hydrogenation of adsorbed $\mathrm{O}$ or $\mathrm{OH}$ species. If $E_{\mathrm{O}}(\varepsilon) \geq-1.09 \mathrm{eV}$, the ratelimiting process is the dissociation of $\mathrm{O}_{2}$. The intercepts are determined as follows: there is an optimal $E_{O}$ value corresponding to the maximum ORR activity (or "volcano top") and our previous work has determined it as $-1.09 \mathrm{eV}^{40}$ The intercept is calculated as $-0.67 \mathrm{eV}$ (and $1.96 \mathrm{eV}$ ) for the left (and right) "volcano leg" so that $E_{\mathrm{a}}$ is minimal (i.e., $0 \mathrm{eV}$ ) when $E_{\mathrm{O}}$ takes the optimal value of $-1.09 \mathrm{eV}$. In the following, we use $E_{O}$ as the descriptor for ORR activity, and drop the subscript for brevity. As indicated in eq 1 , the descriptor $E$ is a function of surface strain $\varepsilon$. Following the microkinetic model of Nørskov et al., ${ }^{39}$ we can express the local ORR reaction rate $r_{i}$ at a surface site $i$ as

$$
r_{i}=f_{0} \mathrm{e}^{-E_{\mathrm{a}}(i) / k_{\mathrm{B}} T}
$$

where $E_{\mathrm{a}}$ is the activation energy defined in eq $1, T$ is temperature, $k_{\mathrm{B}}$ is the Boltzmann constant, and $f_{0}$ is the attempt frequency. The overall ORR reaction rate on a given core/shell NP is thus given by

$$
r_{\mathrm{NP}}=\frac{1}{A} \sum_{i} r_{i}
$$

summing up all local reaction sites and normalized by the total surface area $A$. Clearly, the surface strain $\varepsilon$ is a key quantity determining the overall ORR activity of the NP. By engineering the size, shape, shell thickness, and alloy composition of Pd core/shell NPs, we can elevate their ORR activities well above that of $\mathrm{Pt}$-the aim of the present work. To reach this goal, we need to know the surface strain distribution on the NPs and the dependence of $E$ on $\varepsilon$. In the following, we determine these two quantities.

Previous studies have shown that the dependence of $E$ on $\varepsilon$ is sensitive to local atomic geometries, ${ }^{40,41}$ and since a NP consists of different facets and edges, we have to determine $E(\varepsilon)$ separately for each of them. However, this is a computationally challenging task for NPs with experimentally relevant dimensions (up to $15 \mathrm{~nm}$ ), and especially for NP edges where structural periodicity is absent. To overcome this challenge, we resort to a multiscale quantum mechanics/ molecular mechanics (QM/MM) method ${ }^{42}$ that was developed recently in our group to tackle NP edges. The validation of the $\mathrm{QM} / \mathrm{MM}$ method and its application to core/shell NPs can be found elsewhere. ${ }^{22,35,36}$ To calculate $E(\varepsilon)$ on flat facets, we can use conventional periodic density functional theory (DFT) simulations with Vienna $\mathrm{Ab}$ initio Simulation Package (VASP). ${ }^{43-45}$

To determine the strain distribution on the NP surface, we use the following equation:

$$
\varepsilon_{i}=\frac{1}{d_{0}^{\mathrm{NN}}}\left(\frac{1}{N_{i}^{\mathrm{NN}}} \sum_{j, j \in\langle i, j\rangle} d_{i j}\right)
$$

where $d_{0}^{\mathrm{NN}}$ is the equilibrium nearest neighbor $(\mathrm{NN})$ distance in the bulk Pd; $N_{i}^{\mathrm{NN}}$ is the number of the NN for site $i ; d_{i j}$ is the distance between sites $i$ and $j$ in the relaxed atomic structure of the NP, and the summation goes over all NN sites for $i$. The strain along the edge can be calculated similarly. In this work, we use molecular statics (MS) simulations with binary embedded-atom-method (EAM) potentials ${ }^{46}$ to determine the relaxed atomic geometries of the NPs.

For a given core/shell NP, the following computational procedure is adopted: (1) Several typical shapes of the NP are considered, and for each shape, we identify relevant facets and edges for ORR. (2) For each facet and edge, we can determine $E(\varepsilon)$ using VASP and QM/MM calculations, respectively. (3) For each shape, we select numerous combinations of different particle sizes, shell thicknesses, and alloy compositions of the core, and we perform inexpensive MS calculations for each combination to evaluate the surface strain distribution on the NP. (4) We then estimate the overall ORR rates using eqs 2 and 3 for each NP. Step 2 involves expensive quantum mechanical calculations, but once the $E(\varepsilon)$ dependence is established, it can be used repeatedly in step 4. The actual screening step could be very fast and applied to diverse core materials as long as the corresponding interatomic potentials are available. In the absence of reliable interatomic potentials, one may be able to resort to finite-element modeling for the strain distribution. ${ }^{27,47}$ Note that the ligand effect such as charge redistribution ${ }^{48}$ is not considered explicitly in this work. 
This is (1) because the ligand effect is negligible for a shell thickness greater than two layers ${ }^{42}$ and (2) due to the desire to simplify the computational screening process. However, the ligand effect could be considered in a later and more refined stage of the screening process.

In the following, we apply the multiscale strategy to Pd core/ shell NPs. Three typical particle shapes are examined here, including truncated octahedron (TO), icosahedron (ICO), and octahedron (OCT) as depicted in Figure 1. More complicated (a) Truncated Octahedron

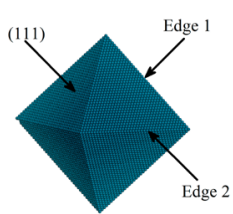

(c) Octahedron

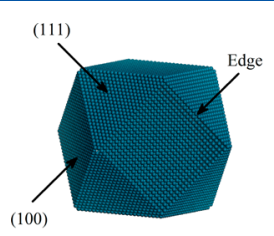

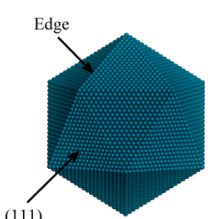

(b) Icosahedron

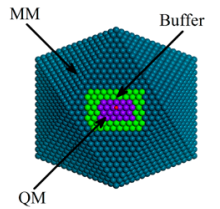

(d) QM/MM Model
Figure 1. Pd-based core/shell NPs in the shape of (a) truncated octahedron, (b) icosahedron, and (c) octahedron, respectively. Various facets and edges are labeled for each shape. (d) Schematic partition of spatial regions in the $\mathrm{QM} / \mathrm{MM}$ model.

shapes can be considered similarly. The truncated octahedron NP consists of $\{111\}$ and $\{100\}$ facets, and $\langle 110\rangle$ edges joining the two facets. The icosahedral NP contains $\{111\}$ facets and some high-index edges. The octahedral NP has $\{111\}$ facets and two types of $\langle 110\rangle$ edges (edge 1 and edge 2$)$. We have performed DFT calculations to obtain $E(\varepsilon)$ on $\{111\}$ and $\{100\}$ facets and QM/MM calculations on the four edges. In this work, we focus on Pd shell with thickness ranging from two to five atomic layers and binary alloy cores. Four different diameters of NPs are considered $(6,8,11$, and $15 \mathrm{~nm})$. Finally, the overall ORR rates for the truncated octahedron (TO), octahedron (OCT), and icosahedron (ISO) NPs are expressed as follows:

$$
\begin{aligned}
r_{\mathrm{TO}} & =\frac{1}{A} \sum_{i, i \in(111)} r_{i}^{(111)}+\frac{1}{A} \sum_{j, j \in(100)} r_{j}^{(100)} \\
+ & \frac{1}{A} \sum_{k, k \in \text { edge }} r_{k}^{\text {edge }} \\
r_{\mathrm{OCT}} & =\frac{1}{A} \sum_{i, i \in(111)} r_{i}^{(111)}+\frac{1}{A} \sum_{j, j \in \text { edge } 1} r_{j}^{\text {edge } 1} \\
+ & \frac{1}{A} \sum_{k, k \in \text { edge } 2} r_{k}^{\text {edge } 2} \\
r_{\mathrm{ICO}} & =\frac{1}{A} \sum_{i, i \in(111)} r_{i}^{(111)}+\frac{1}{A} \sum_{j, j \in \text { edge }} r_{j}^{\text {edge }}
\end{aligned}
$$

In eqs $5-7$, the rate on each facet or edge is obtained via eq 2 . For instance, on the (111) facet, $r_{i}^{(111)}=f_{0} \exp \left[-E_{\mathrm{a}, i}^{(111)} / k_{\mathrm{B}} T\right]$ and the other rates are calculated similarly, where $E_{\mathrm{a}, i}$ is calculated according to eq 1 . As pointed out by Chen et al., ${ }^{49,50}$ the coverage of the spectators and/or intermediates could be important to an accurate determination of the reaction rates. However, the appropriate treatment of the coverage effect would require more sophisticated kinetic modeling, which is beyond the scope of the present paper. Note that the coverage of the intermediates could affect the surface strain, and thus can be partially captured in the present multiscale framework.

\section{COMPUTATIONAL DETAILS}

The molecular statics (MS) calculations are performed using the LAMMPS package ${ }^{51}$ with the EAM potential developed by Zhou et al. ${ }^{46}$ The number of atoms in the MS calculations ranges from $\sim 12000$ for the smallest NPs to $\sim 270000$ for the
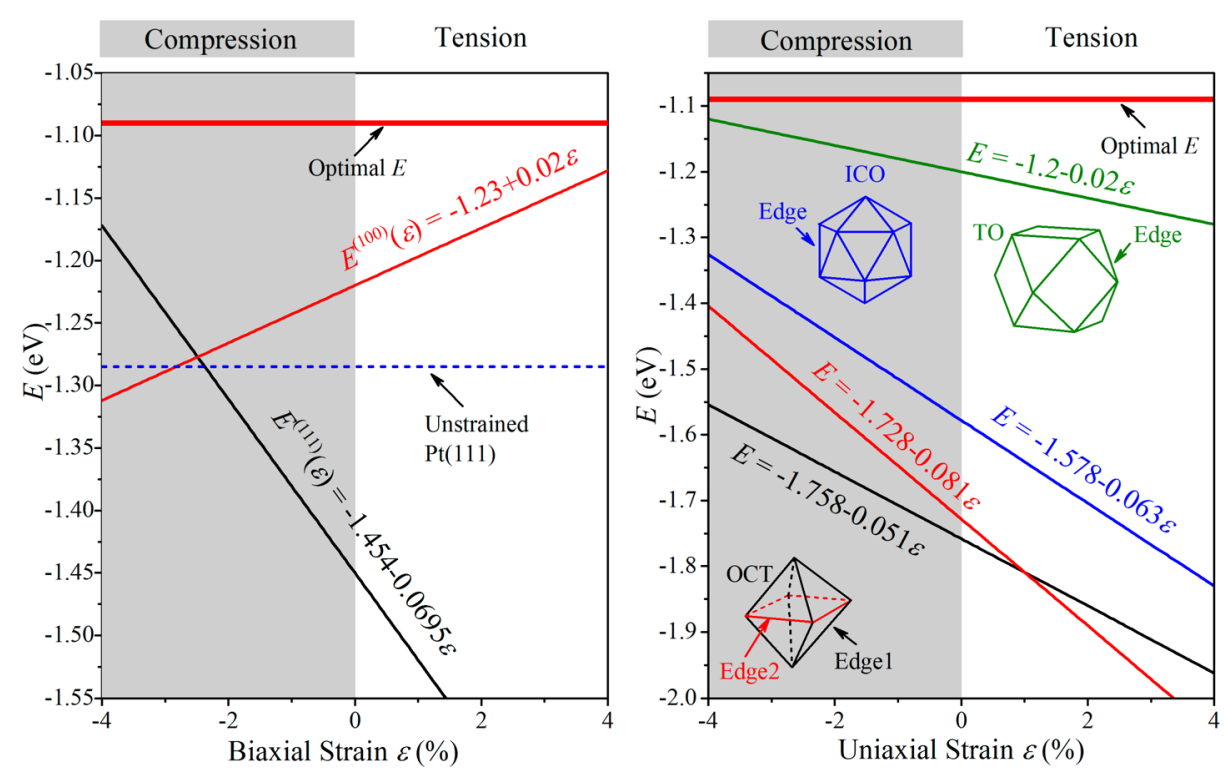

Figure 2. Oxygen adsorption energy $E$ as a function of surface strain $\varepsilon$ on different facets (left) and edges (right). The (red) solid and (blue) dashed horizontal lines represent the optimal $E$ value for maximal ORR activity and the $E$ value on $\mathrm{Pt}(111)$ surface, respectively. The insets of the right panel indicate the corresponding edges. 

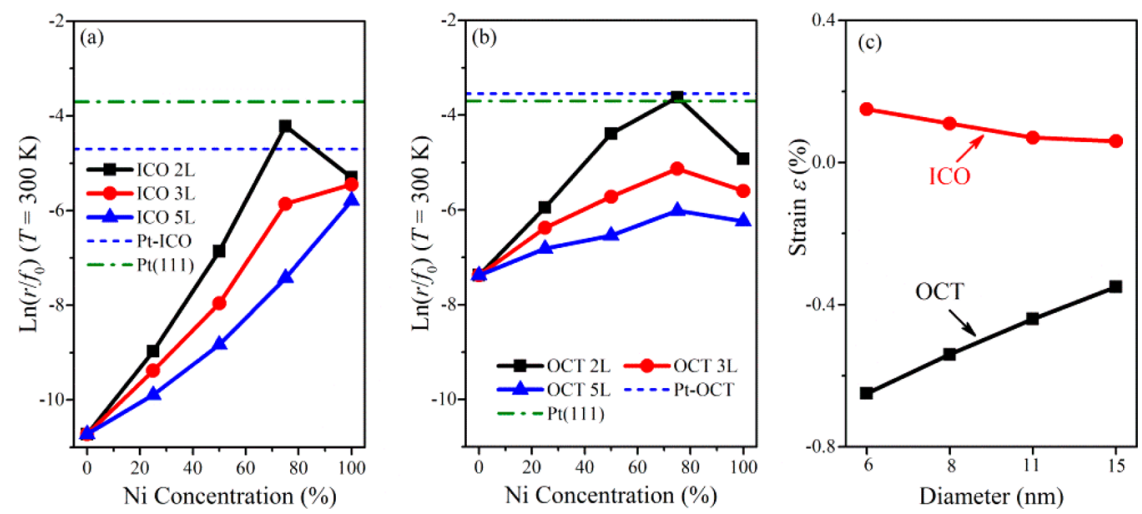

Figure 3. Logarithm of the ORR reaction rate as a function of Ni concentration for (a) icosahedral NPs and (b) octahedral NPs, respectively. The diameter of the NP is $8 \mathrm{~nm}$ and the Pd shell thickness ranges from two layers $(2 \mathrm{~L})$ to three layers $(3 \mathrm{~L})$ and five layers $(5 \mathrm{~L})$. The blue horizontal line represents the ORR rate for the pure Pt NP in the icosahedral (a) and octahedral (b) shapes. The green horizontal line indicates the ORR rate on pure $\operatorname{Pt}(111)$ surface. (c) Average surface strain on the pure Pd icosahedral and octahedral NP as a function of its diameter.

largest NPs. The equilibrium lattice constant of Pd is $3.893 \AA$. DFT calculations are carried out to evaluate the oxygen adsorption energy on flat surfaces with VASP. ${ }^{43-45}$ The Perdew-Burke-Ernzerhof exchange-correlation functional ${ }^{52}$ and projected-augmented-wave pseudopotential ${ }^{53}$ are employed in the VASP calculations. The energy cutoff is $400 \mathrm{eV}$ and the Fermi-Dirac smearing width is $0.02 \mathrm{eV}$. The atomic geometries are considered fully relaxed when the force on each unconstrained atom is less than $0.02 \mathrm{eV} / \AA$. For the $(111)$ surface, we have used a four-layer $4 \times 3$ surface slab and a $4 \times 5$ $\times 1$ Monkhorst-Pack (MP) $k$-mesh for the integrations in the Brillouin zone. ${ }^{54}$ For the (100) surface, we have used a fourlayer $4 \times 4$ slab and a $4 \times 4 \times 1 \mathrm{MP} k$-mesh. The vacuum thickness is chosen as $15 \AA$ for both surfaces, which has been shown to yield converged adsorption energy. The QM/MM simulations are performed to calculate the oxygen adsorption energy on the edges of the NPs. The NP is divided into three regions in the $\mathrm{QM} / \mathrm{MM}$ calculations: $\mathrm{QM}, \mathrm{MM}$, and buffer regions. The technical details of the $\mathrm{QM} / \mathrm{MM}$ method can be found in our previous publications. ${ }^{35,42}$ As shown in Figure $1 \mathrm{~d}$, the combined QM and buffer regions consist of about 300-400 DFT atoms, and the MM region has between $\sim 12000$ and 270000 EAM atoms, depending on the NP size and shape. The atomic relaxation is carried out until the force on each atom is less than $0.02 \mathrm{eV} / \AA$.

\section{RESULTS AND DISCUSSION}

4.1. $E(\varepsilon)$ on Different Facets and Edges. To determine $E(\varepsilon)$, biaxial strain ranging from $-4 \%$ (compression) to $4 \%$ (tension) is applied to the (100) and (111) surfaces of Pd. The oxygen adsorption energy $E$ is calculated as a function of the strain $\varepsilon$, along with the optimal $E$ value and $E$ value for the unstrained, flat $\mathrm{Pt}(111)$ surface, shown in Figure 2. The closer the energy $E$ to the optimal value, the higher the ORR activity. First, we find that, under strains, both $\operatorname{Pd}(111)$ and $\operatorname{Pd}(100)$ facets can be more active than the Pt(111) surface. Thus, Pd core/shell NPs could potentially overtake Pt as highly active ORR catalysts. The second and more striking observation is that the (111) and (100) facets have opposite $E(\varepsilon)$ dependence. On the closely packed (111) facet, surface compression is found to enhance the ORR activity while the opposite is true on the (100) facet. More importantly, the (100) surface is more active than the (111) surface as long as the surface compression is less than $2.6 \%$ in magnitude. In particular, when stretched, the (100) facet could become highly active, approaching the maximum ORR activity. Since the (111) surface is dominant in face-centered-cubic metals, such as $\mathrm{Pt}$ and $\mathrm{Pd}$, surface compression is widely known to promote ORR activity. However, if one can access the (100) facet, surface tension can be equally or even more beneficial to the ORR activity. The combination and competition of the two could have profound impacts on the design of ORR catalysts. Lastly, the ORR activity on the (111) surface is more sensitive-with a larger slope-to the strain than that on the (100) surface. To understand the opposite strain dependence between the (100) and (111) facets, we calculate the density of states of the two surfaces with $4 \%$ biaxial compression. As shown in Figure S1 in the Supporting Information, we find that the d-band centers of the (100) and (111) facets shift in opposite directions relative to the Fermi energy. We believe that the opposite shifts of the d-band center underscore their opposite strain dependence.

Uniaxial strains are applied to the edges of the NPs, and the $\mathrm{QM} / \mathrm{MM}$ calculations are performed to calculate the oxygen adsorption energy on the edges as a function of the strain shown in the right panel of Figure 2. Interestingly, all edges exhibit the same trend, i.e., the more the compression, the higher the ORR activity. Among the three NP shapes, the edge of the truncated octahedron displays the highest ORR activity while the edges of the octahedron exhibit the lowest activity, irrespective of the strain. The edge of the truncated octahedron is equivalent to the (211) stepped surface, which has been shown to yield higher $E$ than the $\operatorname{Pd}(111)$ surface, ${ }^{2,41}$ consistent with our result. The ORR activity on the edge of the truncated octahedron is the least sensitive to the strain, however.

Since the icosahedral and octahedral NPs have only (111) facets while the truncated octahedron has both (111) and (100) facets, we divide them into two groups in the following discussion: the icosahedron and octahedron NPs in one group and the truncated octahedron NPs in another. A common technique for synthesizing core/shell NPs involves acid leaching of the non-noble-metal component in the shell and then a thermal treatment to form a noble-metal skin. This motivates us to focus on $\mathrm{Pd}-\mathrm{M}$ alloys as core materials ( $\mathrm{M}$ is an element yet to be determined). With the $\mathrm{Pd}-\mathrm{M}$ alloys, one can leach out $\mathrm{M}$ to form a $\mathrm{Pd}$ shell and leave $\mathrm{Pd}-\mathrm{M}$ as the core. In the following, we will examine a combination of size, shape, shell thickness, and chemical composition ( $\mathrm{M}$ and its 
concentration) for Pd-M@Pd NPs to achieve higher ORR activity than Pt.

4.2. Icosahedral and Octahedral Core/Shell NPs. As a preliminary screening step, we select an icosahedral core/shell NP with diameter $d=8 \mathrm{~nm}$ and shell thickness of three layers. The core is a random binary alloy with a chemical composition of $\mathrm{M}_{50} \mathrm{Pd}_{50}(\mathrm{M}=\mathrm{Ag}, \mathrm{Al}, \mathrm{Au}, \mathrm{Co}, \mathrm{Cu}, \mathrm{Fe}, \mathrm{Mo}, \mathrm{Ni}, \mathrm{Pd}, \mathrm{Pt}, \mathrm{Ta}$, $\mathrm{Ti})$. These elements are chosen because their EAM potentials are available for MS calculations. We then perform the MS simulations to estimate the strain at the center of the (111) facet, and the results are summarized in Figure S2. As mentioned earlier, a compressive strain can enhance ORR activity on both the (111) facet and all edges of the NPs. Therefore, it is a reasonable starting point by choosing a core material that yields compressive surface strains to narrow down the choice of alloy compositions. Thus, we pick $\mathrm{M}=\mathrm{Ni}$ because it produces the greatest compressive strain of $3 \%$ as indicated in Figure S2. In the following, we will focus on icosahedral and octahedral $\mathrm{Ni}_{x} \mathrm{Pd}_{1-x} @ \mathrm{Pd}$ core/shell NPs.

Figure 3 presents the logarithm of the ORR reaction rate $\left[\ln \left(r / f_{0}\right)\right]$ on $\mathrm{Ni}_{x} \mathrm{Pd}_{1-x} @ \mathrm{Pd} \mathrm{NPs}$ as a function of $\mathrm{Ni}$ concentration $x$ and shell thickness at $300 \mathrm{~K}$. We find that the icosahedral and octahedral NPs exhibit similar trends (up to $75 \%$ of $\mathrm{Ni}$ ): the higher the $\mathrm{Ni}$ concentration, the higher the ORR rate. For the same Ni concentration, the thinner the shell, the higher the ORR activity. These trends can be understood from the perspective of surface strain. Since Ni has a much smaller (9.5\%) lattice constant than Pd, a higher concentration of Ni yields a greater compression, hence a higher ORR activity. A thinner shell can more easily transmit the strain to the surface, and thus is more beneficial to the ORR activity. More importantly, for the same shape, the core/shell NPs with $75 \%$ $\mathrm{Ni}$ concentration and thin shells (two layers) could yield comparable or higher ORR activities than the pure Pt NPs. Moreover, the octahedral $\mathrm{Ni}_{75} \mathrm{Pd}_{25} @ \mathrm{Pd} \mathrm{NP}$ exhibits higher ORR activity than the flat $\mathrm{Pt}(111)$ surface. These results suggest that core materials with smaller lattice constants than $\mathrm{Pd}$, such as $\mathrm{Cu}$ and $\mathrm{Co}$, may also lead to superior ORR activities to pure Pt.

For the same Ni concentration and shell thickness, we find that the octahedral NPs are more active than the icosahedral NPs except those with pure Ni cores, as shown in Figure 3. To understand this observation, we calculate the surface strain on a pure Pd NP $(d=8 \mathrm{~nm})$ in the octahedral and icosahedral shapes. As found in Figure 3c, the icosahedral NP has an intrinsic built-in surface tension while the octahedral NP has an intrinsic built-in surface compression. The similar built-in strains have also been observed by others. ${ }^{27,55}$ Thus, the intrinsic compression of the octahedral NP combined with the compression induced by the Ni core further enhances the ORR activity. On the contrary, the compression induced by $\mathrm{Ni}$ is partially canceled by the intrinsic tension, leading to less net compression on the icosahedral NP for the same Ni concentration and shell thickness. These results highlight the importance of the particle shape on ORR activity.

We note that, for very thin shells, there is a kink or even a dip in the ORR rates for $75<x<100$. This is due to undesirable plastic deformation-dislocations are nucleated on the NP surface as shown in Figure 4, which relaxes the surface compression and lowers the ORR activity.

To examine the size dependence of ORR activity, we select $\mathrm{Ni}_{50} \mathrm{Pd}_{50} @ \mathrm{Pd}$ as an example. As shown in the upper panels of Figure 5, the icosahedral NP displays a monotonic increase of

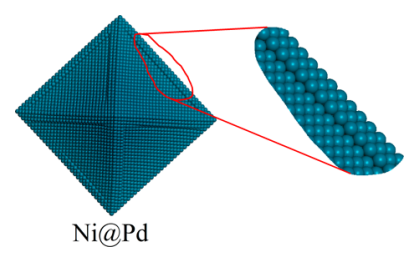

Figure 4. Atomistic model of Ni@Pd octahedral NP $(d=8 \mathrm{~nm})$. The shell thickness is two layers. The inset is a blown-up view near the NP edge, showing the formation of dislocations.

ORR activity as its diameter, irrespective of its shell thickness. On the other hand, a nonmonotonic size dependence is observed for the octahedral NP with shell thicknesses of $3 \mathrm{~L}$ and $5 \mathrm{~L}$; for a thickness of $2 \mathrm{~L}$, it recovers the monotonic size dependence. The size dependences of the octahedral NP can be understood from the surface strain induced by its core, presented in the lower panels of Figure 5. Note that in Figure 5 the intrinsic strain has been subtracted from the total strain to arrive at the core-induced strain; this is necessary because the intrinsic strain has a monotonic dependence on the diameter. For the $\mathrm{Ni}_{50} \mathrm{Pd}_{50}$ core, the induced strain exhibits a nonmonotonic size dependence for the thicker shells (3L and $5 \mathrm{~L}$ ) and a monotonic dependence for the thinnest shell $(2 \mathrm{~L})$, which tracks the ORR rates precisely. The monotonic size dependence appears prevalent for other alloy compositions. Our results demonstrate the interplay between the materials factors and the complexity of the materials design-the optimal size of the core/shell NPs depends on factors such as NP shape, shell thickness, and chemical composition in a complicated manner.

4.3. Truncated Octahedron Core/Shell NPs. Comparing to the icosahedron and the octahedron counterparts, the truncated octahedron NPs are more complex because they consist of both (111) and (100) facets, which have opposite strain dependence of $E$. To illustrate the interplay between the two facets, we choose $\mathrm{Ni}_{x} \mathrm{Pd}_{1-x}$ and $\mathrm{Ag}_{x} \mathrm{Pd}_{1-x}$ as core materials because $\mathrm{Ni}_{50} \mathrm{Pd}_{50}$ and $\mathrm{Ag}_{50} \mathrm{Pd}_{50}$ yield the highest surface compression and tension, respectively, among the 12 elements examined in Figure S2. The ORR rates for $\mathrm{Ni}_{x} \mathrm{Pd}_{1-x} @ \mathrm{Pd}$ and $\mathrm{Ag}_{x} \mathrm{Pd}_{1-x} @ \mathrm{Pd}$ core/shell NPs at $300 \mathrm{~K}$ are presented in Figure 6 along with those of the pure Pt NP in the truncated octahedron shape and the $\operatorname{Pt}(111)$ surface. First, we note that the pure Pt NP in the truncated octahedron shape outperforms the other two shapes by very large margins. As shown in Figure 7 (black lines), the intrinsic built-in strains on both (111) and (100) facets of pure truncated octahedron NPs are compressive $(-0.7$ to $-0.8 \%)$. For this compression, the ORR activity on the edge of the truncated octahedron is much higher than that on the edges of the icosahedron and octahedron. Furthermore, the ORR activity on the (100) facet is much higher than that on the (111) facet. Since the truncated octahedron have both (111) and (100) facets while the icosahedron and octahedron have only the (111) facet, the pure Pd NPs in the truncated octahedron shape are predicted to be more active than the other two shapes, as observed in Figure 6.

For the core/shell NPs, we find that, in the same $\mathrm{Pd}$ concentration, $\mathrm{Ag}_{x} \mathrm{Pd}_{1-x} @ \mathrm{Pd} \mathrm{NPs}$ are always more active than $\mathrm{Ni}_{x} \mathrm{Pd}_{1-x} @ \mathrm{Pd} \mathrm{NPs}$ regardless of the shell thickness and the particle size. However, the two behave quite differently with respect to the chemical composition. For $\mathrm{Ag}_{x} \mathrm{Pd}_{1-x} @ \mathrm{Pd}$, the ORR rate increases monotonically with the $\mathrm{Ag}$ concentration, but for $\mathrm{Ni}_{x} \mathrm{Pd}_{1-x} @ \mathrm{Pd}$, the rate dips for intermediate $\mathrm{Ni}$ concentrations. Moreover, $\mathrm{Ag}_{x} \mathrm{Pd}_{1-x} @ \mathrm{Pd}$ NPs with shell 

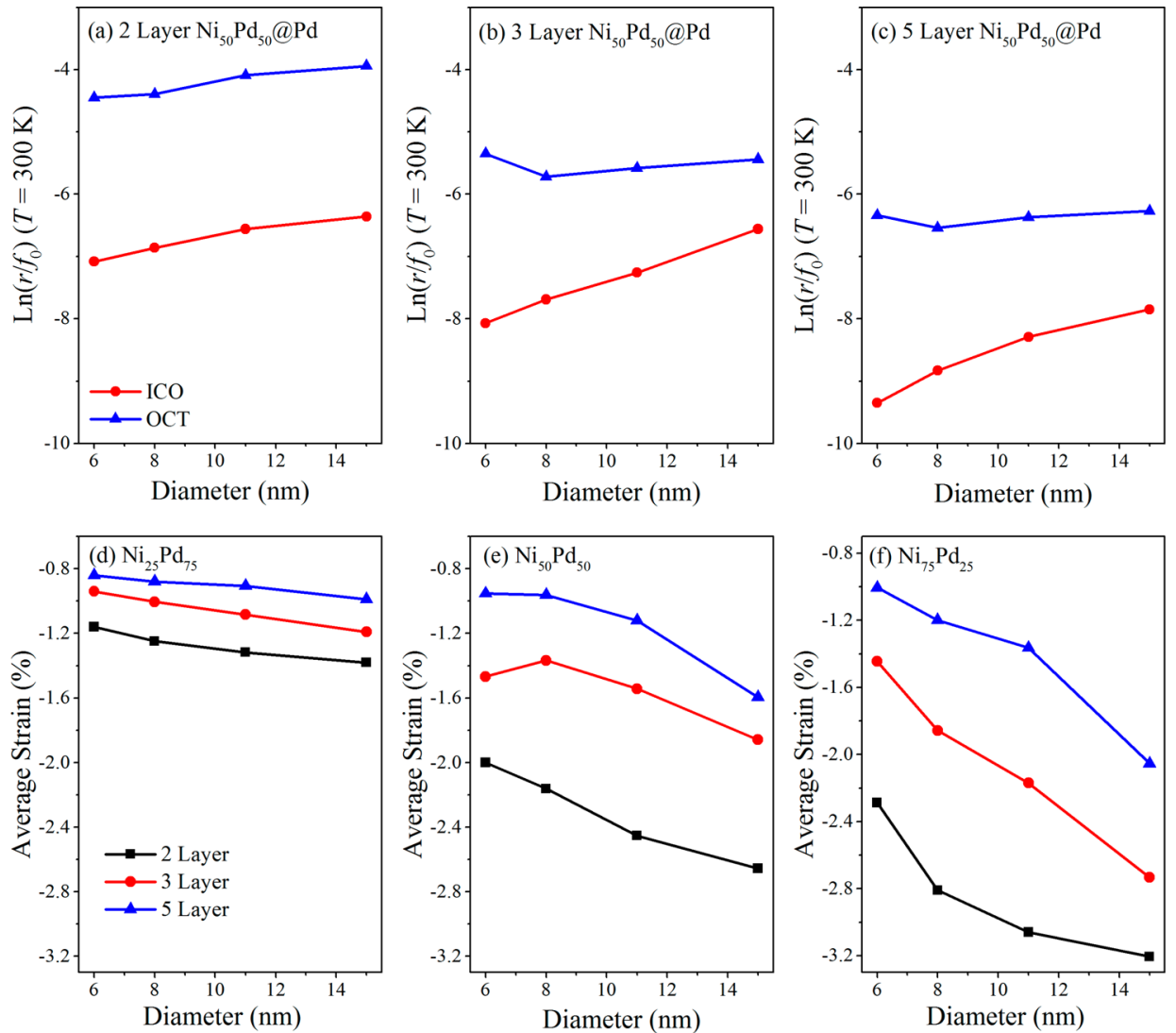

Figure 5. (a)-(c) Logarithm of ORR reaction rate as a function of diameter for $\mathrm{Ni}_{50} \mathrm{Pd}_{50} @ \mathrm{Pd} \mathrm{NP}$ with a shell thickness of two, three, and five layers, respectively. (d)-(f) Average surface strain as a function of diameter induced by $\mathrm{Ni}_{25} \mathrm{Pd}_{75}, \mathrm{Ni}_{50} \mathrm{Pd}_{50}$, and $\mathrm{Ni}_{75} \mathrm{Pd}_{25}$ cores, respectively.
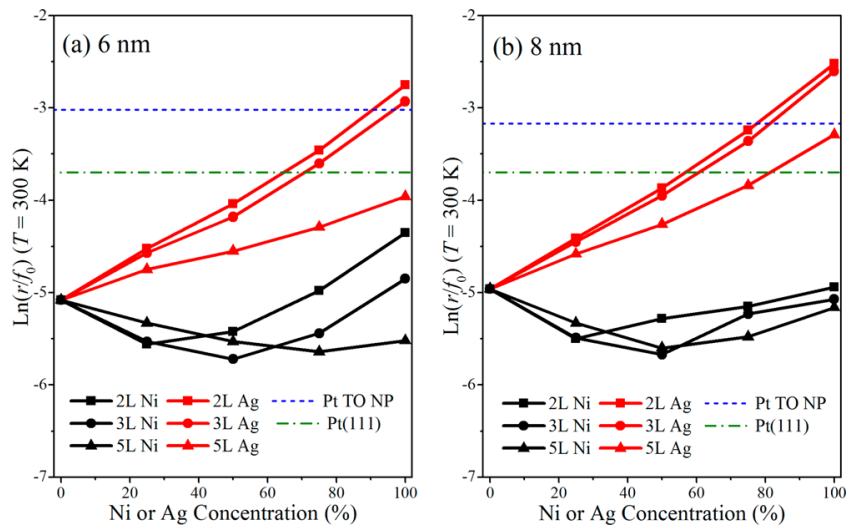

Figure 6. Logarithm of ORR reaction rate as a function of $\mathrm{Ni}$ or $\mathrm{Ag}$ concentration for truncated octahedron NP with diameters of (a) 6 and (b) $8 \mathrm{~nm}$. Black and red lines represent the rates for $\mathrm{Ni}_{x} \mathrm{Pd}_{1-x} @ P d$ and $\mathrm{Ag}_{x} \mathrm{Pd}_{1-x} @ \mathrm{Pd} \mathrm{NP}$, respectively. The blue and green horizontal lines indicate the ORR rates for truncated octahedron pure Pt NP and pure $\operatorname{Pt}(111)$ surface, respectively.

thicknesses of two and three layers display similar ORR rates, higher than that of the five-layer shell. More importantly, the ORR activity of both $\mathrm{Ag}_{x} \mathrm{Pd}_{1-x} @ \mathrm{Pd}$ and $\mathrm{Ni}_{x} \mathrm{Pd}_{1-x} @ \mathrm{Pd} \mathrm{NPs}$ could exceed that of the $\operatorname{Pt}(111)$ surface, and for a large $x$, $\mathrm{Ag}_{x} \mathrm{Pd}_{1-x} @ \mathrm{Pd} \mathrm{NPs}$ exhibit higher ORR activity than the pure $\mathrm{Pt}$ NPs in the same truncated octahedron shape. Finally, the $\mathrm{Ag}_{x} \mathrm{Pd}_{1-x} @ \mathrm{Pd} \mathrm{NPs}$ are predicted to be more active than the $\mathrm{Pt}(111)$ surface for almost all $x$ values.

To shed light into the ORR rate dependence on chemical composition, we calculate the average surface strain of
$\mathrm{Ag}_{x} \mathrm{Pd}_{1-x} @ \mathrm{Pd}$ and $\mathrm{Ni}_{x} \mathrm{Pd}_{1-x} @ \mathrm{Pd} \mathrm{NPs}$ as a function of their diameter and alloy composition, summarized in Figure 7. For $\mathrm{Ag}_{x} \mathrm{Pd}_{1-x} @ \mathrm{Pd} \mathrm{NPs}$, we note that most compositions yield tensile strains, and the higher the Ag concentration, the larger the surface tension. For most compositions, the larger the NP, the greater the surface tension. In addition, the tension on the (100) facet is stronger than on the (111) facet. The overall reaction rate is dominated by more active sites due to its exponential dependence on $E$ or $\varepsilon$. As a result, the enhanced ORR activity on the more active (100) facet overcompensates the reduced ORR activity on the less active (111) facet, thanks to the surface tension. Thus, the overall ORR activity would increase as a function of the surface tension. Therefore, we conclude that the ORR activity of $\mathrm{Ag}_{x} \mathrm{Pd}_{1-x} @ \mathrm{Pd}$ NPs should increase as a function of the particle size and $\mathrm{Ag}$ concentration, both evident in Figure 6. The situation of $\mathrm{Ni}_{x} \mathrm{Pd}_{1-x} @ \mathrm{Pd} \mathrm{NPs}$ is more complicated. The ORR rates first decrease and then increase as a function of $\mathrm{Ni}$ concentration, and the turning point depends on the shell thickness and NP size. This behavior originates from the competition between the (111) and (100) facets. Alloying of $\mathrm{Ni}$ introduces compression on both the (111) and (100) facets, and the enhanced ORR activity on the (111) facet is offset by the reduced ORR activity on the (100) facet. For Ni concentration below 25\%, both facets experience compressions, which are less than $2.8 \%$, and as a result, the ORR activity is dominated by the (100) facet. Since the compression is detrimental to the ORR activity on the (100) facet, the overall activity of the NP is suppressed as the $\mathrm{Ni}$ concentration increases from 0 to $25 \%$, as shown in Figure 6. Moreover, the NP with the thickest shell (five layers) tends to have smaller compression, thus higher ORR activity, as 

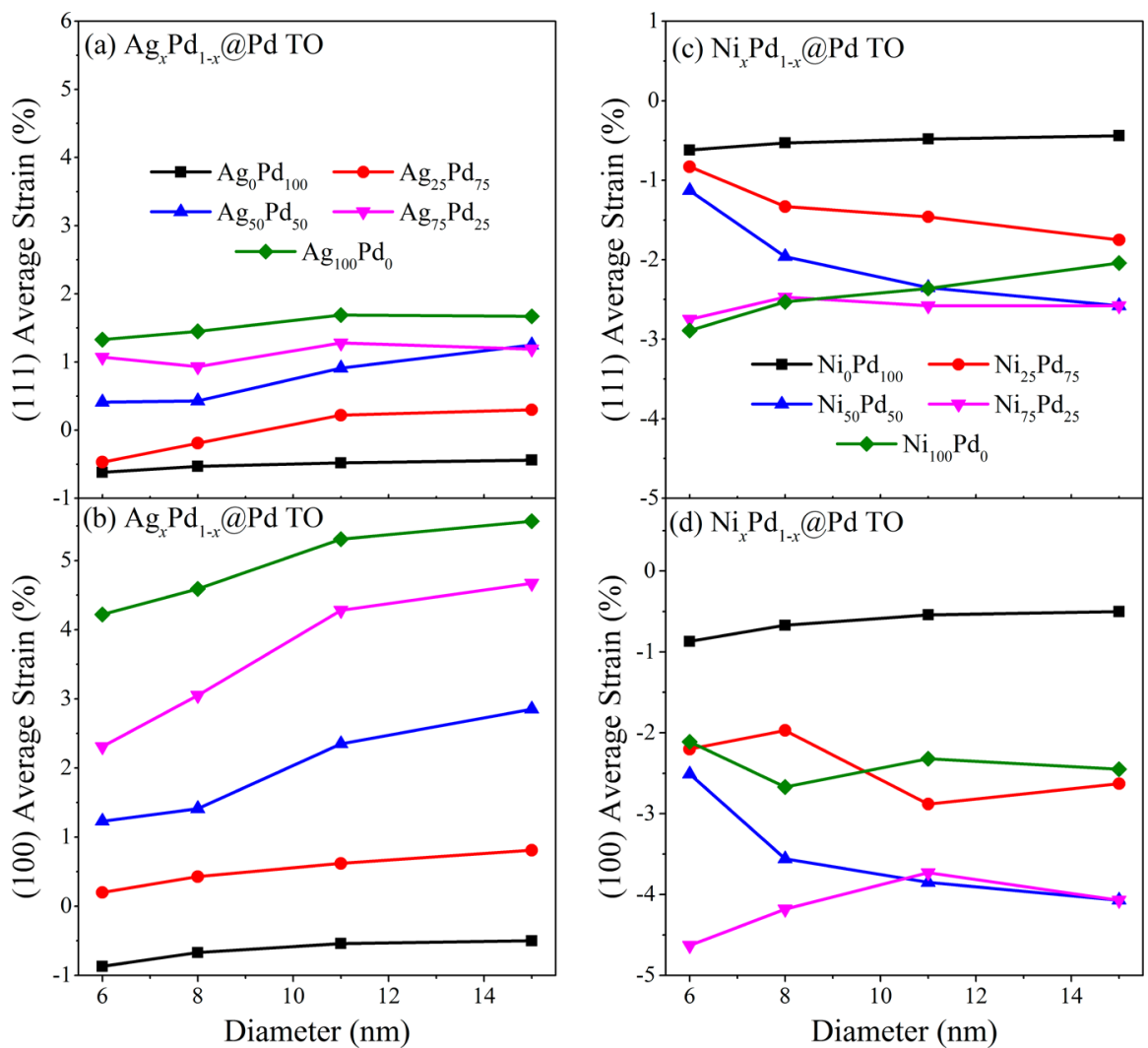

Figure 7. Average surface strain as a function of diameter on (a) (111) facet of $\mathrm{Ag}_{x} \mathrm{Pd}_{1-x} @ \mathrm{Pd} \mathrm{NP},(\mathrm{b})(100)$ facet of $\mathrm{Ag}_{x} \mathrm{Pd}_{1-x} @ \mathrm{Pd} \mathrm{NP},(\mathrm{c})(111)$ facet of $\mathrm{Ni}_{x} \mathrm{Pd}_{1-x} @ \mathrm{Pd} \mathrm{NP}$, and (d) (100) facet of $\mathrm{Ni}_{x} \mathrm{Pd}_{1-x} @ \mathrm{Pd} \mathrm{NP}$. The Pd shell thickness is three layers.
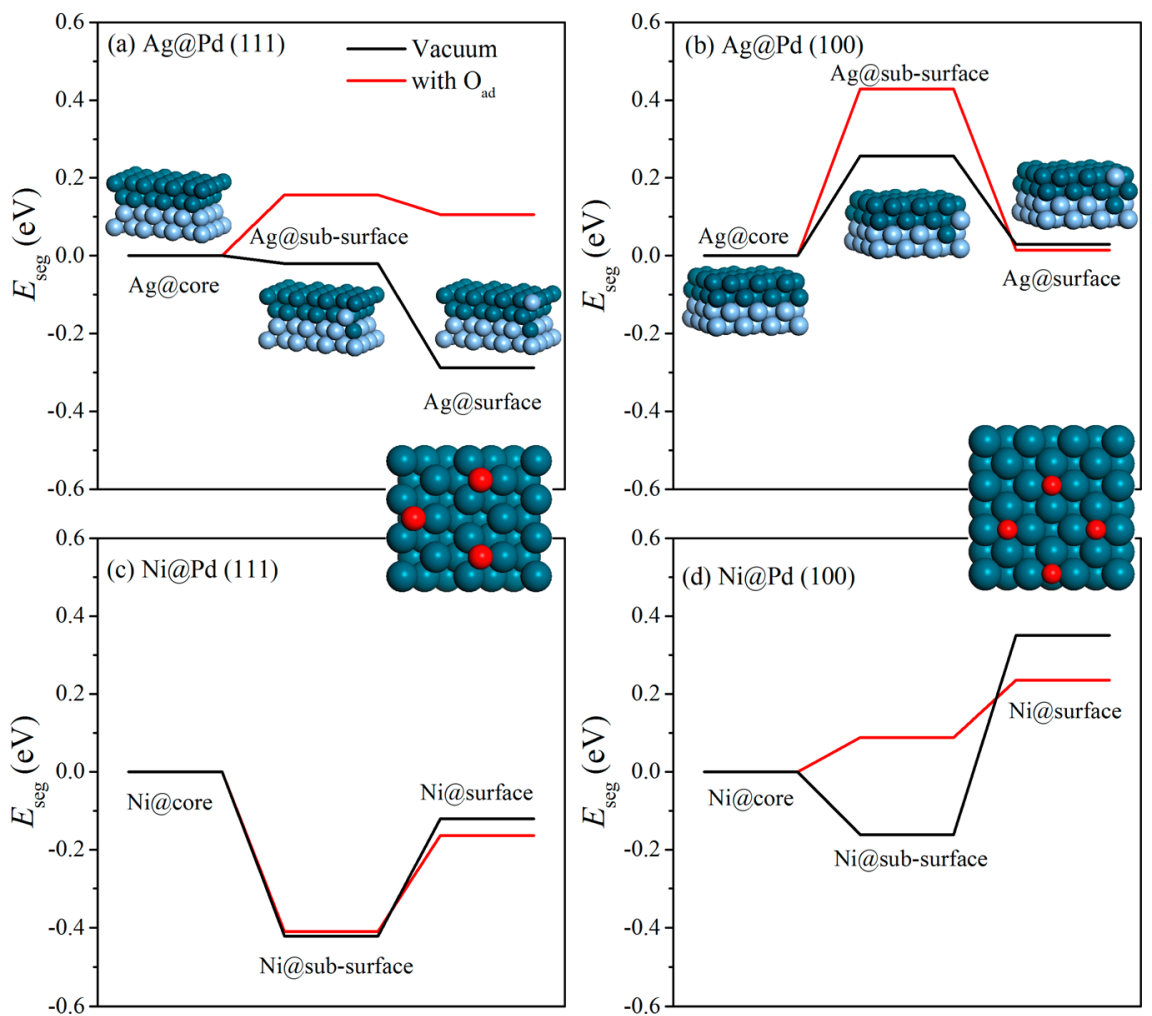

Figure 8. Segregation energy $E_{\text {seg }}$ for (a) (111) surface of Ag@Pd, (b) (100) surface of Ag@Pd, (c) (111) surface of Ni@Pd, and (d) (100) surface of Ni@Pd. The red and black lines represent $E_{\text {seg }}$ with and without $\mathrm{O}$ adatoms $\left(\mathrm{O}_{\mathrm{ad}}\right)$, respectively. The atomistic models for the surfaces are shown in insets. The red and cyan spheres represent $\mathrm{O}$ and $\mathrm{Pd}$ atoms, respectively. 
shown in Figure 6. As the Ni concentration increases from 25 to $50 \%$, so is the surface compression, which exceeds $2.8 \%$. As a result, the (111) facet overtakes (100) as the dominant surface for ORR activity. Since compression is beneficial to ORR activity on the dominating (111) facet, the overall ORR activity increases as a function of $\mathrm{Ni}$ concentration. The only exception to this trend is the NP with the thickest shell, whose turning point occurs at a higher $\mathrm{Ni}$ concentration. Finally, as $\mathrm{Ni}$ concentration exceeds $70 \%$, plastic deformation takes place, similar to the octahedral NPs. The nucleation of dislocations on the (100) facet relaxes the elastic strain and its compression becomes smaller, which enhances the ORR activity on the facet. The strain on the (111) facet is not affected. Overall, we find that the truncated octahedron NPs are more active than the icosahedral and octahedral NPs. In particular, we predict that Ag@Pd core/shell NPs with 2-3 layers of Pd shell and 6-8 $\mathrm{nm}$ in diameter exhibit the highest ORR activity, well above that of the flat $\mathrm{Pt}(111)$ surface or Pt NPs.

4.4. Stability of Core/Shell NPs. In addition to catalytic activity, stability is another crucial property that has to be considered in the design of core/shell NPs. Unfortunately, unlike ORR activity, there is no generally accepted descriptor to assess the stability of ORR catalysts. Previous studies have examined relative surface energies and binding energies of component elements for this purpose. ${ }^{40,56,57}$ Here, we follow an approach used by Zhang and Henkelman where segregation energy, $E_{\text {seg }}$, is employed as a measure for surface stability. ${ }^{38} E_{\text {seg }}$ is defined as the energy required to swap a core atom with its adjacent shell atom, and a positive $E_{\text {seg }}$ indicates that the swap is thermodynamically unfavorable. To simplify the calculations, we focus on the (111) and (100) facets with two layers of Pd shell and either Ag or Ni core, representing Ag@Pd and Ni@ $\mathrm{Pd}$ core/shell NPs. We then exchange a core atom $(\mathrm{Ag}$ or $\mathrm{Ni})$ with its neighboring Pd atom on either the subsurface or the surface. $E_{\text {seg }}$ is calculated in both a vacuum and an oxygen-rich environment. The former is modeled with a clean surface, while the latter is modeled with adsorbed $\mathrm{O}$ atoms on the surface. Specifically, the $\mathrm{O}$ coverage is $\theta_{\mathrm{O}}=25 \%$ on the (111) surface and $\theta_{\mathrm{O}}=22 \%$ on the $(100)$ surface as shown in Figure 8 .

For the $\mathrm{Ag} @ \mathrm{Pd}(111)$ facet, we find that, in a vacuum, the core/shell NP is not thermodynamically stable as $\mathrm{Ag}$ atoms prefer to segregate to the $\operatorname{Pd}(111)$ surface and subsurface. However, in the oxygen-rich environment, the segregation is suppressed at the subsurface. Our results agree with that of Zhang and Henkelman ${ }^{58}$ and experiment. ${ }^{59}$ On the (100) facet, there are very high energy barriers for $\mathrm{Ag}$ segregation to the subsurface in both a vacuum and an oxygen-rich environment. Thus, Ag@Pd NPs are expected to be thermodynamically stable in ORR, and the (100) facet is more stable than the (111) facet. For the Ni@Pd(111) facet, although Ni may segregate to the subsurface in both environments, it is thermodynamically unfavorable to be on the surface. Similarly, $\mathrm{Ni}$ segregation to the (100) surface is also suppressed in both environments. Therefore, we conclude that $\mathrm{Ag}_{x} \mathrm{Pd}_{1-x} @ \mathrm{Pd}$ and $\mathrm{Ni}_{x} \mathrm{Pd}_{1-x} @ \mathrm{Pd} \mathrm{NPs}$ with high $\mathrm{Ag}$ and $\mathrm{Ni}$ concentrations are thermodynamically stable in the oxygen-rich environment. Note that the electrochemical stability is also important for the design of core/shell NPs, but this is beyond the scope of the present work.

\section{CONCLUSION}

In summary, we have proposed a multiscale computational framework to design core/shell NPs for ORR. Central to the framework are the linear scaling relations between the oxygen adsorption energy $E$ and the surface strain $\varepsilon$, which are determined for the (111) and (100) facets and four edges from DFT and QM/MM calculations, respectively. While the surface compression is found to enhance ORR activity on the (111) facet and all edges, the opposite is true on the (100) facet. Based on the linear scaling relations and the microkinetic model, we can estimate the ORR rate as a function of surface strain on core/shell NPs, with the latter determined by the MS calculations. Using the multiscale approach, we have systematically examined the ORR activity on Pd-based core/shell NPs as a function of their shape, size, shell thickness, and alloy composition of the core. Three NP shapes are explored, and the truncated octahedron is found to be most active among them. For the NPs in the icosahedral and octahedral shapes, we predict that $\mathrm{Ni}_{x} \mathrm{Pd}_{1-x} @ \mathrm{Pd} \mathrm{NPs}$ with high $\mathrm{Ni}$ concentrations and thinner shells could be more active than the flat $\mathrm{Pt}(111)$ surface and/or Pt NPs in the same shape. The octahedron shape is more active than the icosahedron, owing to the intrinsic compression in the former and tension in the latter. For the NPs in the truncated octahedron shape, we find that $\mathrm{Ag}_{x} \mathrm{Pd}_{1-x} @ \mathrm{Pd}$ and $\mathrm{Ni}_{x} \mathrm{Pd}_{1-x} @ \mathrm{Pd} \mathrm{NPs}$ with high $\mathrm{Ag}$ and $\mathrm{Ni}$ concentrations could be more active than the flat $\operatorname{Pt}(111)$ surface. $\mathrm{Ag}_{x} \mathrm{Pd}_{1-x} @ \mathrm{Pd} \mathrm{NPs}$ are more active than $\mathrm{Ni}_{x} \mathrm{Pd}_{1-x} @ \mathrm{Pd}$ NPs under the same conditions, and with high $\mathrm{Ag}$ concentrations, $\mathrm{Ag}_{x} \mathrm{Pd}_{1-x} @ \mathrm{Pd} \mathrm{NPs}$ are predicted to be the most active among all core/shell NPs considered here. The thermodynamic stability of the core/shell NPs is examined, and the highly active $\mathrm{Ni}_{x} \mathrm{Pd}_{1-x} @ \mathrm{Pd}$ and $\mathrm{Ag}_{x} \mathrm{Pd}_{1-x} @ \mathrm{Pd} \mathrm{NPs}$ are found to be thermodynamically stable.

\section{ASSOCIATED CONTENT}

\section{Supporting Information}

The Supporting Information is available free of charge on the ACS Publications website at DOI: 10.1021/acs.jpcc.6b11337.

Shift of d-band center for $\operatorname{Pd}(111)$ and $\operatorname{Pd}(100)$ surfaces under different biaxial strains; results of preliminary screening of NP core alloys (PDF)

\section{AUTHOR INFORMATION}

\section{Corresponding Author}

*E-mail: ganglu@csun.edu. Tel.: 818-677-2021.

ORCID 우

Zhengzheng Chen: 0000-0002-4911-9649

Notes

The authors declare no competing financial interest.

\section{ACKNOWLEDGMENTS}

We acknowledge valuable discussions with William A. Curtin, Yurui Gao, Zhonglong Zhao, and Amir Pirayandeh. This work was supported by the U.S. Army Research Office through Grant W911NF-11-1-0353.

\section{REFERENCES}

(1) Greeley, J.; Stephens, I. E. L.; Bondarenko, A. S.; Johansson, T. P.; Hansen, H. A.; Jaramillo, T. F.; Rossmeisl, J.; Chorkendorff, I.; Nørskov, J. K. Alloys of Platinum and Early Transition Metals as Oxygen Reduction Electrocatalysts. Nat. Chem. 2009, 1, 552-556.

(2) Peterson, A.; Grabow, L.; Brennan, T.; Shong, B.; Ooi, C.; Wu, D.; Li, C. W.; Kushwaha, A.; Medford, A.; Mbuga, F.; et al. Finite-Size Effects in $\mathrm{O}$ and $\mathrm{CO}$ Adsorption for the Late Transition Metals. Top. Catal. 2012, 55, 1276-1282. 
(3) Lv, H.; Xi, Z.; Chen, Z.; Guo, S.; Yu, Y.; Zhu, W.; Li, Q.; Zhang, X.; Pan, M.; Lu, G.; et al. A New Core/Shell NiAu/Au Nanoparticle Catalyst with Pt-like Activity for Hydrogen Evolution Reaction. J. Am. Chem. Soc. 2015, 137, 5859-5862.

(4) Zhang, L.; Iyyamperumal, R.; Yancey, D. F.; Crooks, R. M.; Henkelman, G. Design of Pt-Shell Nanoparticles with Alloy Cores for the Oxygen Reduction Reaction. ACS Nano 2013, 7, 9168-9172.

(5) Tsai, H. C.; Hsieh, Y. C.; Yu, T. H.; Lee, Y. J.; Wu, Y. H.; Merinov, B. V.; Wu, P. W.; Chen, S. Y.; Adzic, R. R.; Goddard, W. A. DFT Study of Oxygen Reduction Reaction on Os/Pt Core-Shell Catalysts Validated by Electrochemical Experiment. ACS Catal. 2015, $5,1568-1580$.

(6) An, W.; Liu, P. Rationalization of $\mathrm{Au}$ Concentration and Distribution in AuNi@Pt Core-Shell Nanoparticles for Oxygen Reduction Reaction. ACS Catal. 2015, 5, 6328-6336.

(7) Alayoglu, S.; Nilekar, A. U.; Mavrikakis, M.; Eichhorn, B. Ru-Pt Core-Shell Nanoparticles for Preferential Oxidation of Carbon Monoxide in Hydrogen. Nat. Mater. 2008, 7, 333-338.

(8) Lim, B.; Jiang, M. J.; Camargo, P. H. C.; Cho, E. C.; Tao, J.; Lu, X. M.; Zhu, Y. M.; Xia, Y. N. Pd-Pt Bimetallic Nanodendrites with High Activity for Oxygen Reduction. Science 2009, 324, 1302-1305.

(9) Shen, L. L.; Zhang, G. R.; Miao, S.; Liu, J.; Xu, B. Q. Core-Shell Nanostructured $\mathrm{Au} @ \mathrm{Ni}_{\mathrm{m}} \mathrm{Pt}_{2}$ Electrocatalysts with Enhanced Activity and Durability for Oxygen Reduction Reaction. ACS Catal. 2016, 6, $1680-1690$

(10) Stamenkovic, V. R.; Fowler, B.; Mun, B. S.; Wang, G. F.; Ross, P. N.; Lucas, C. A.; Marković, N. M. Improved Oxygen Reduction Activity on $\mathrm{Pt}_{3} \mathrm{Ni}(111)$ via Increased Surface Site Availability. Science 2007, 315, 493-497.

(11) Guo, S.; Zhang, S.; Sun, S. H. Tuning Nanoparticle Catalysis for the Oxygen Reduction Reaction. Angew. Chem., Int. Ed. 2013, 52, $8526-8544$.

(12) Tang, W. J.; Zhang, L.; Henkelman, G. Catalytic Activity of Pd/ $\mathrm{Cu}$ Random Alloy Nanoparticles for Oxygen Reduction. J. Phys. Chem. Lett. 2011, 2, 1328-1331.

(13) Zhang, L.; Henkelman, G. Computational Design of AlloyCore@Shell Metal Nanoparticle Catalysts. ACS Catal. 2015, 5, 655660.

(14) Guo, S.; Zhang, S.; Sun, S. H. Tuning Nanoparticle Catalysis for the Oxygen Reduction Reaction. Angew. Chem., Int. Ed. 2013, 52, $8526-8554$.

(15) Yu, W.; Porosoff, M. D.; Chen, J. G. Review of Pt-Based Bimetallic Catalysis: From Model Surfaces to Supported Catalysts. Chem. Rev. 2012, 112, 5780-5817.

(16) Wang, C.; Daimon, H.; Onodera, T.; Koda, T.; Sun, S. H. A General Approach to the Size- and Shape-Controlled Synthesis of Platinum Nanoparticles and Their Catalytic Reduction of Oxygen. Angew. Chem., Int. Ed. 2008, 47, 3588-3591.

(17) Tian, N.; Zhou, Z. Y.; Sun, S. G.; Ding, Y.; Wang, Z. L. Synthesis of Tetrahexahedral Platinum Nanocrystals with High-Index Facets and High Electro-Oxidation Activity. Science 2007, 316, 732-735.

(18) Zhu, H.; Zhang, S.; Guo, S.; Su, D.; Sun, S. H. Synthetic Control of FePtM Nanorods $(\mathrm{M}=\mathrm{Cu}, \mathrm{Ni})$ to Enhance the Oxygen Reduction Reaction. J. Am. Chem. Soc. 2013, 135, 7130-7133.

(19) Gong, M. G.; Jin, X.; Sakidja, R.; Ren, S. Q. Synergistic Strain Engineering Effect of Hybrid Plasmonic, Catalytic, and Magnetic Core-Shell Nanocrystals. Nano Lett. 2015, 15, 8347-8353.

(20) Zhang, S. B.; Zunger, A. Surface-Reconstruction-Enhanced Solubility of N, P, As, and Sb in III-V Semiconductors. Appl. Phys. Lett. 1997, 71, 677-679.

(21) Zhu, R.; Pan, E.; Chung, P. W. Fast Multiscale Kinetic Monte Carlo Simulations of Three-Dimensional Self-Assembled Quantum Dot Islands. Phys. Rev. B: Condens. Matter Mater. Phys. 2007, 75, 205339.

(22) Zhang, S.; Zhang, X.; Jiang, G.; Zhu, H.; Guo, S.; Su, D.; Lu, G.; Sun, S. H. Tuning Nanoparticle Structure and Surface Strain for Catalysis Optimization. J. Am. Chem. Soc. 2014, 136, 7734-7739.

(23) Mavrikakis, M.; Hammer, B.; Nørskov, J. K. Effect of Strain on the Reactivity of Metal Surfaces. Phys. Rev. Lett. 1998, 81, 2819-2822.
(24) Xu, Y.; Ruban, A. V.; Mavrikakis, M. Adsorption and Dissociation of $\mathrm{O}_{2}$ on Pt-Co and Pt-Fe Alloys. J. Am. Chem. Soc. 2004, 126, 4717-4725.

(25) Strasser, P.; Koh, S.; Anniyev, T.; Greeley, J.; More, K.; Yu, C.; Liu, Z.; Kaya, S.; Nordlund, D.; Ogasawara, H.; et al. Lattice-Strain Control of the Activity in Dealloyed Core-Shell Fuel Cell Catalysts. Nat. Chem. 2010, 2, 454-460.

(26) Kitchin, J. R.; Nørskov, J. K.; Barteau, M. A.; Chen, J. G. Role of Strain and Ligand Effects in the Modification of the Electronic and Chemical Properties of Bimetallic Surfaces. Phys. Rev. Lett. 2004, 93, 156801 .

(27) Moseley, P.; Curtin, W. A. Computational Design of Strain in Core-Shell Nanoparticles for Optimizing Catalytic Activity. Nano Lett. 2015, 15, 4089-4095.

(28) Guo, S. J.; Zhang, X.; Zhu, W. L.; He, K.; Su, D.; MendozaGarcia, A.; Ho, S. F.; Lu, G.; Sun, S. H. Nanocatalyst Superior to Pt for Oxygen Reduction Reactions: The Case of Core/Shell $\mathrm{Ag}(\mathrm{Au}) / \mathrm{CuPd}$ Nanoparticles. J. Am. Chem. Soc. 2014, 136, 15026-15033.

(29) Wang, C.; Waje, M.; Wang, X.; Tang, J. M.; Haddon, R. C.; Yan, Y. Proton Exchange Membrane Fuel Cells with Carbon Nanotube Based Electrodes. Nano Lett. 2004, 4, 345-348.

(30) Cui, C.; Gan, L.; Heggen, M.; Rudi, S.; Strasser, P. Compositional Segregation in Shaped Pt Alloy Nanoparticles and Their Structural Behaviour during Electrocatalysis. Nat. Mater. 2013, $12,765-771$.

(31) Cui, C.; Li, H.; Liu, X.; Gao, M.; Yu, S. Surface Composition and Lattice Ordering-Controlled Activity and Durability of $\mathrm{CuPt}$ Electrocatalysts for Oxygen Reduction Reaction. ACS Catal. 2012, 2, 916-924.

(32) Ji, X.; Lee, K.; Holden, R.; Zhang, L.; Zhang, J.; Botton, G.; Couillard, M.; Nazar, L. Nanocrystalline Intermetallics on Mesoporous Carbon for Direct Formic Acid Fuel Cell Anodes. Nat. Chem. 2010, 2, 286-293.

(33) Kibler, L. A.; El-Aziz, A. M.; Hoyer, R; Kolb, D. M. Tuning Reaction Rates by Lateral Strain in a Palladium Monolayer. Angew. Chem., Int. Ed. 2005, 44, 2080-2084.

(34) Jiang, G.; Zhu, H. Y.; Zhang, X.; Shen, B.; Wu, L. H.; Zhang, S.; Lu, G.; Wu, Z. B.; Sun, S. H. Core/Shell Face-Centered Tetragonal FePd/Pd Nanoparticles as an Efficient Non-Pt Catalyst for the Oxygen Reduction Reaction. ACS Nano 2015, 9, 11014-11022.

(35) Zhang, X.; Lu, G. Computational Design of Core/Shell Nanoparticles for Oxygen Reduction Reactions. J. Phys. Chem. Lett. 2014, 5, 292-297.

(36) Nørskov, J. K.; Bligaard, T.; Logadottir, A.; Bahn, S. R.; Hansen, L. B.; Bollinger, M. V.; Bengaard, H. S.; Hammer, B.; Sljivancanin, Z.; Mavrikakis, M.; et al. Universality in Heterogeneous Catalysis. J. Catal. 2002, 209, 275-278

(37) Bligaard, T.; Nørskov, J. K.; Dahl, S.; Matthiesen, J.; Christensen, C. H.; Sehested, J. The Brønsted-Evans-Polanyi Relation and the Volcano Curve in Heterogeneous Catalysis. J. Catal. 2004, 224, 206-217.

(38) Stamenkovic, V. R.; Mun, B. S.; Mayrhofer, K. J. J.; Ross, P. N.; Markovic, N. M.; Rossmeisl, J.; Greeley, J.; Nørskov, J. K. Changing the Activity of Electrocatalysts for Oxygen Reduction by Tuning the Surface Electronic Structure. Angew. Chem., Int. Ed. 2006, 45, 28972901.

(39) Nørskov, J. K.; Rossmeisl, J.; Logadottir, A.; Lindqvist, L.; Kitchin, J. R.; Bligaard, T.; Jónsson, H. Origin of the Overpotential for Oxygen Reduction at a Fuel-Cell Cathode. J. Phys. Chem. B 2004, 108, 17886-17892.

(40) Chen, Z.; Yang, Y.; Kumar, S.; Lu, G. First-Principles Prediction of Oxygen Reduction Activity on Pd-Cu-Si Metallic Glasses. J. Phys. Chem. C 2014, 118, 28609-28615.

(41) Mittendorfer, F.; Seriani, N.; Dubay, O.; Kresse, G. Morphology of Mesoscopic Rh and Pd Nanoparticles under Oxidizing Conditions. Phys. Rev. B: Condens. Matter Mater. Phys. 2007, 76, 233413.

(42) Zhang, X.; Lu, G.; Curtin, W. A. Multiscale Quantum/Atomistic Coupling Using Constrained Density Functional Theory. Phys. Rev. B: Condens. Matter Mater. Phys. 2013, 87, 054113. 
(43) Kresse, G.; Hafner, J. Ab Initio Molecular Dynamics for Liquid Metals. Phys. Rev. B: Condens. Matter Mater. Phys. 1993, 47, 558.

(44) Kresse, G.; Hafner, J. Ab Initio Molecular-Dynamics Simulation of the Liquid-Metal-Amorphous-Semiconductor Transition in Germanium. Phys. Rev. B: Condens. Matter Mater. Phys. 1994, 49, 1425114269 .

(45) Kresse, G.; Furthmüller, J. Efficiency of Ab-Initio Total Energy Calculations for Metals and Semiconductors Using a Plane-Wave Basis Set. Comput. Mater. Sci. 1996, 6, 15-50.

(46) Zhou, X.; Johnson, R; Wadley, H. Misfit-Energy-Increasing Dislocations in Vapor-Deposited CoFe/NiFe Multilayers. Phys. Rev. B: Condens. Matter Mater. Phys. 2004, 69, 144113.

(47) Langlois, C.; Benzo, P.; Arenal, R.; Benoit, M.; Nicolai, J.; Combe, N.; Ponchet, A.; Casanove, M. J. Fully Crystalline Faceted Fe$\mathrm{Au}$ Core-Shell Nanoparticles. Nano Lett. 2015, 15, 5075-5080.

(48) Tang, W. J.; Henkelman, G. Charge Redistribution in Core-Shell Nanoparticles to Promote Oxygen Reduction. J. Chem. Phys. 2009, 130, 194504.

(49) Yang, F.; Zhang, Q. F.; Liu, Y. W.; Chen, S. L. A Theoretical Consideration on the Surface Structure and Nanoparticle Size Effects of Pt in Hydrogen Electrocatalysis. J. Phys. Chem. C 2011, 115, 19311-19319.

(50) Chen, J. X.; Luo, S. W.; Liu, Y. W.; Chen, S. L. Theoretical Analysis of Electrochemical Formation and Phase Transition of Oxygenated Adsorbates on Pt(111). ACS Appl. Mater. Interfaces 2016, 8, 20448-20458.

(51) Plimpton, S. J. Fast Parallel Algorithms for Short-Range Molecular Dynamics. J. Comput. Phys. 1995, 117, 1-19 ( http:// lammps.sandia.gov).

(52) Perdew, J. P.; Burke, K.; Ernzerhof, M. Generalized Gradient Approximation Made Simple. Phys. Rev. Lett. 1996, 77, 3865-3868.

(53) Kresse, G.; Joubert, D. From Ultrasoft Pseudopotentials to the Projector Augmented-Wave Method. Phys. Rev. B: Condens. Matter Mater. Phys. 1999, 59, 1758-1775.

(54) Monkhorst, H. J.; Pack, J. D. SpeApplications cial Points for Brillouin-Zone Integrations. Phys. Rev. B 1976, 13, 5188-5192.

(55) Wu, J.; Qi, L.; You, H.; Gross, A.; Li, J.; Yang, H. Icosahedral Platinum Alloy Nanocrystals with Enhanced Electrocatalytic Activities. J. Am. Chem. Soc. 2012, 134, 11880-11883.

(56) Ferrando, R.; Jellinek, J.; Johnston, R. L. Nanoalloys: From Theory to of Alloy Clusters and Nanoparticles. Chem. Rev. 2008, 108, 845-910.

(57) Wang, L. L.; Johnson, D. D. Predicted Trends of Core-Shell Preferences for 132 Late Transition-Metal Binary-Alloy Nanoparticles. J. Am. Chem. Soc. 2009, 131, 14023-14029.

(58) Zhang, L.; Henkelman, G. Tuning the Oxygen Reduction Activity of Pd Shell Nanoparticles with Random Alloy Cores. J. Phys. Chem. C 2012, 116, 20860-20865.

(59) Weir, M. G.; Knecht, M. R.; Frenkel, A. I.; Crooks, R. M. Structural Analysis of PdAu Dendrimer-Encapsulated Bimetallic Nanoparticles. Langmuir 2010, 26, 1137-1146. 